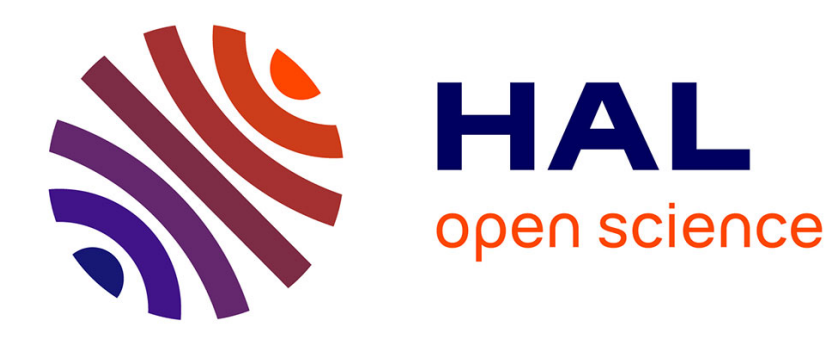

\title{
A note on biharmonic submanifolds of product spaces Julien Roth
}

\section{- To cite this version:}

Julien Roth. A note on biharmonic submanifolds of product spaces. Journal of Geometry, 2013, 104

(2), pp.375-381. hal-00827336v2

\section{HAL Id: hal-00827336 \\ https://hal.science/hal-00827336v2}

Submitted on 12 Jun 2013

HAL is a multi-disciplinary open access archive for the deposit and dissemination of scientific research documents, whether they are published or not. The documents may come from teaching and research institutions in France or abroad, or from public or private research centers.
L'archive ouverte pluridisciplinaire HAL, est destinée au dépôt et à la diffusion de documents scientifiques de niveau recherche, publiés ou non, émanant des établissements d'enseignement et de recherche français ou étrangers, des laboratoires publics ou privés. 


\title{
A note on biharmonic submanifolds of product spaces
}

\author{
Julien ROTH
}

Mathematics Subject Classification (2010). 53C42, 53C43.

Keywords. biharmonic submanifolds, product spaces.

\begin{abstract}
We investigate biharmonic submanifolds of the product of two space forms. We prove a necessary and sufficient condition for biharmonic submanifolds in these product spaces. Then, we obtain mean curvature estimates for proper-biharmonic submanifold of a product of two unit spheres. We also prove a non-existence result in the case of the product of a sphere and a hyperbolic space.
\end{abstract}

\section{Introduction}

A harmonic map $\psi$ between two Riemannian manifolds $(M, g)$ and $(N, h)$ is defined as a critical point of the energy functional

$$
E(\psi)=\frac{1}{2} \int_{M}|d \psi|^{2} d v_{g} .
$$

In [4], Eells and Sampson proposed a natural generalization of harmonic maps. The map $\psi$ is said to be biharmonic if it is a critical point of the bienergy functional

$$
E_{2}(\psi)=\frac{1}{2} \int_{M}|\tau(\psi)|^{2} d v_{g},
$$

where $\tau(\psi)=\operatorname{tr}(\nabla d \psi)$ is the tension field which vanishes for harmonic map. The Euler-Lagrange equation for the bienergy functional is given by $\tau_{2}(\psi)=0$ (see [8]), where $\tau_{2}(\psi)$ is the bitension field and is defined by

$$
\tau_{2}(\psi)=\Delta \tau(\psi)-\operatorname{tr}\left(R^{N}(d \psi, \tau(\psi)) d \psi\right) .
$$

Here, $\Delta$ stands for the rough Laplacian acting on sections of $\psi^{-1}(T N)$ and $R^{N}$ is the curvature tensor of $N$.

Note that we use the following sign conventions for the Laplacian and the curavture tensor. We set

$$
\Delta V=\operatorname{tr}\left(\nabla^{2} V\right) \quad \text { and } \quad R^{N}(X, Y)=\left[\nabla_{X}^{N}, \nabla_{Y}^{N}\right]-\nabla_{[X, Y]}^{N} .
$$


or any $V \in \Gamma\left(\psi^{-1}(T N)\right)$ and $X, Y \in \Gamma(T N)$.

Since any harmonic map is biharmonic, we will focus on biharmonic maps that are not harmonic, which are called proper-biharmonic.

A biharmonic submanifold of a Riemannian manifold is a submanifold for which the inclusion map is biharmonic.

Biharmonic submanifolds of different ambient spaces have been intensively studied in the last decade, in particular for real space forms [2], complex space forms [5], Sasakian space forms [6] or the product of a sphere with the real line [7]. In the present paper, we investigate the biharmonic submanifolds of product manifolds. Precisely, we will consider submanifolds into the products of two real space forms, with a focus on the case of the product of two spheres, or the product of a sphere with a hyperbolic space.

\section{Preliminaries}

Let $\left(M^{m}, g\right)$ be a Riemannian manifold isometrically immersed into the product space $\left(\mathbb{M}^{n_{1}}\left(c_{1}\right) \times \mathbb{M}^{n_{2}}\left(c_{2}\right), \widetilde{g}\right)$, where $\mathbb{M}^{n_{j}}\left(c_{j}\right)$ is the $n_{j}$-dimensional real space form of curvature $c_{j}$, for $j=1,2$. We denote by $F$ the product structure of $\mathbb{M}^{n_{1}}\left(c_{1}\right) \times \mathbb{M}^{n_{2}}\left(c_{2}\right)$. Namely, the map $F: T \mathbb{M}^{n_{1}}\left(c_{1}\right) \times T \mathbb{M}^{n_{2}}\left(c_{2}\right) \longrightarrow$ $T \mathbb{M}^{n_{1}}\left(c_{1}\right) \times T \mathbb{M}^{n_{2}}\left(c_{2}\right)$ is defined by $F\left(X_{1}+X_{2}\right)=X_{1}-X_{2}$, where $X_{j} \in$ $T \mathbb{M}^{n_{j}}\left(c_{j}\right)$. Obviously, $F$ satisfies

$$
\begin{gathered}
F^{2}=I d \quad(\text { and } F \neq I d), \\
\widetilde{g}(F X, Y)=\widetilde{g}(X, F Y), \\
\widetilde{\nabla} F=0 .
\end{gathered}
$$

Moreover, we recall that the curvature tensor of $\left(\mathbb{M}^{n_{1}}\left(c_{1}\right) \times \mathbb{M}^{n_{2}}\left(c_{2}\right), \widetilde{g}\right)$ is given by

$$
\begin{aligned}
\widetilde{R}(X, Y) Z= & a[\langle Y, Z\rangle X-\langle X, Z\rangle Y+\langle F Y, Z\rangle F X-\langle F X, Z\rangle F Y] \\
& +b[\langle Y, Z\rangle F X-\langle X, Z\rangle F Y+\langle Y, F Z\rangle X-\langle X, F Z\rangle Y](, 4)
\end{aligned}
$$

with $a=\frac{c_{1}+c_{2}}{2}$ and $b=\frac{c_{1}-c_{2}}{2}$. The product structure $F$ induces the existence of the following four operators

$$
f: T M \longrightarrow T M, h: T M \longrightarrow N M, s: N M \longrightarrow T M \text { and } t: N M \longrightarrow N M
$$

defined for any $X \in T M$ and $\xi \in N M$ by

$$
F X=f X+h X \quad \text { and } \quad F \xi=s \xi+t \xi .
$$


From Equations (1) and (2), we deduce that $f$ and $t$ are symmetric and we have the following relations between these four operators

$$
\begin{aligned}
& f^{2} X=X-s h X, \\
& t^{2} \xi=\xi-h s \xi, \\
& f s \xi+s t \xi=0, \\
& h f X+t h X=0, \\
& \widetilde{g}(h X, \xi)=\widetilde{g}(X, s \xi),
\end{aligned}
$$

for any $X \in \Gamma(T M)$ and $\xi \in \Gamma(N M)$.

We have now all the ingredients to state our results.

\section{Main results}

First, we give a necessary and sufficient condition characterizing biharmonicity in product spaces.

Theorem 3.1. Let $M$ be a submanifold of $\mathbb{M}^{n_{1}}\left(c_{1}\right) \times \mathbb{M}^{n_{2}}\left(c_{2}\right)$ of dimension $m$. Then $M$ is biharmonic if and only if the two following equations hold

$$
\begin{gathered}
-\Delta^{\perp} H+\operatorname{tr} B\left(\cdot, A_{H} \cdot\right)=a[m H-h s H+\operatorname{tr}(f) t H]+b[m t H+\operatorname{tr}(f)(H] 1) \\
\frac{m}{2} \operatorname{grad}|H|^{2}+2 \operatorname{tr} A_{\nabla^{\perp} H}(\cdot)=a[-f s H+\operatorname{tr}(f) s H]+b(m-1) s H .(12)
\end{gathered}
$$

Proof. It is a classical fact (see [1, 3, 7] for instance) that, after decomposition into tangential and normal parts, $\tau_{2}(\psi)=0$ is equivalent to

$$
\left\{\begin{array}{l}
-\Delta^{\perp} H+\operatorname{tr} B\left(\cdot, A_{H^{\cdot}}\right)+\operatorname{tr}(\widetilde{R}(\cdot, H) \cdot)^{\perp}=0 \\
\frac{m}{2} \operatorname{grad}|H|^{2}+2 \operatorname{tr} A_{\nabla^{\perp} H}(\cdot)+2 \operatorname{tr}(\widetilde{R}(\cdot, H) \cdot)^{\top}=0 .
\end{array}\right.
$$

From the expression of the curvature tensor $\widetilde{R}$, we get

$$
\begin{aligned}
\operatorname{tr}(\widetilde{R}(\cdot, H) \cdot)= & a\left[-m H+F\left((F H)^{\top}\right)-\operatorname{tr}(f) F H\right] \\
& +b\left[-m F H+(F H)^{\top}-\operatorname{tr}(f) H\right] .
\end{aligned}
$$

Now, we use the decomposition of $F$ into the 4 operators $f, h, s$ and $t$ to obtain

$$
\begin{aligned}
\operatorname{tr}(\widetilde{R}(\cdot, H) \cdot)= & a[-m H+f s H+h s H-\operatorname{tr}(f) s H-\operatorname{tr}(f) t H] \\
& +b[-m s H-m t H+s H-\operatorname{tr}(f) H] .
\end{aligned}
$$

By identification of the tangential and normal parts, we get the two equations of the theorem.

In particular, we have the following particular cases. 
Corollary 3.2. Let $M$ a submanifold of $\mathbb{M}^{n_{1}}\left(c_{1}\right) \times \mathbb{M}^{n_{2}}\left(c_{2}\right)$ of dimension $m$.

1. If FH is tangent to $M$, then $M$ is biharmonic if and only if

$$
\left\{\begin{array}{l}
-\Delta^{\perp} H+\operatorname{tr} B\left(\cdot, A_{H} \cdot\right)-[a(m-1)+b \operatorname{tr}(f)] H=0, \\
\frac{m}{2} \operatorname{grad}|H|^{2}+2 \operatorname{tr} A_{\nabla^{\perp} H}(\cdot)+[a \operatorname{tr}(f)-b(m-1)] F H=0 .
\end{array}\right.
$$

2. If $F H$ is normal to $M$, then $M$ is biharmonic if and only if

$$
\left\{\begin{array}{l}
-\Delta^{\perp} H+\operatorname{tr} B\left(\cdot, A_{H} \cdot\right)-[a m+b \operatorname{tr}(f)] H-[b m+a \operatorname{tr}(f)] F H=0, \\
\frac{m}{2} g r a d|H|^{2}+2 \operatorname{tr} A_{\nabla^{\perp} H}(\cdot)=0 .
\end{array}\right.
$$

Proof. If $F H$ is tangent, then by the definition of the operators $s$ and $t$, we have $s H=F H$ and $t H=0$. Hence, by (7), we have $h s H=H$ and we get the equations of Theorem 3.1.

Now, if $F H$ is normal, then $s H=0$ and $t H=F H$ and we conclude immediately from Theorem 3.1.

From now on, we will focus on the case where the ambient space is a product of two spheres of same radius. Note that in this case, we have $a=\frac{c_{1}+c_{2}}{2}=c_{1}$ and $b=\frac{c_{1}-c_{2}}{2}=0$. For the sake of simplicity, we will assume that the common radius is 1 . First, we have an estimate for the length of the mean curvature vector when $F H$ is tangent to the submanifold.

Corollary 3.3. Let $M$ be a submanifold of $\mathbb{S}^{p} \times \mathbb{S}^{n-p}$, of dimension $m \geqslant 2$ with non-zero constant mean curvature and such that $F H$ is tangent. Then we have

1. If $M$ is proper-biharmonic, then $0<|H|^{2} \leqslant \frac{m-1}{m}$.

2. If $|H|^{2}=\frac{(m-1)}{m}$, then $M$ is proper-biharmonic if and only if it is pseudo-umbilical, $\nabla^{\perp} H=0$ and $\operatorname{tr}(f)=0$.

Proof. Let $M$ be a biharmonic submanifold of $\mathbb{S}^{p} \times \mathbb{S}^{n-p}$ with non-zero constant mean curvature. Since we assume that $F H$ is tangent, by the first assertion of Corollary 3.2, we have

$$
-\Delta^{\perp} H+\operatorname{tr} B\left(\cdot, A_{H} \cdot\right)-(m-1) H=0 .
$$

Hence, by taking the scalar product with $H$, we have

$$
-\left\langle\Delta^{\perp} H, H\right\rangle=(m-1)|H|^{2}-\left|A_{H}\right|^{2} .
$$

Using the Bochner formula and the fact that $|H|$ is constant, we get

$$
(m-1)|H|^{2}=\left|A_{H}\right|^{2}+\left|\nabla^{\perp} H\right|^{2} .
$$

Since $A_{H}$ is the shape operator associated with the mean curvature, by Cauchy-Schwarz inequality, we get $\left|A_{H}\right|^{2} \geqslant m|H|^{4}$. Therefore, we have

$$
(m-1)|H|^{2} \geqslant m|H|^{4}+\left|\nabla^{\perp} H\right|^{2} \geqslant m|H|^{4} .
$$


Since $|H|$ is a non-zero constant, we have $0<|H|^{2} \leqslant \frac{m-1}{m}$.

Now, if $|H|^{2}=\frac{(m-1)}{m}$, if $M$ is proper-biharmonic, then the inequalities above become equalities. First, we have $\nabla^{\perp} H=0$. Moreover, equality occurs in the Cauchy-Schwarz inequality $\left|A_{H}\right|^{2} \geqslant m|H|^{4}$, that is, $M$ is pseudoumbilical. Finally since $\nabla^{\perp} H=0$, the second equation in Corollary 3.2 becomes $\operatorname{tr}(f) F H=0$. Since $H$ has constant positive norm and since $F$ is an isometry, then $F H$ vanishes nowhere. Hence, we have $\operatorname{tr}(f)=0$.

Conversly, if $M$ is pseudo-umbilcal, with $\nabla^{\perp} H=0$ and $\operatorname{tr}(f)=0$, then, we have obviously

$$
\left\{\begin{array}{l}
-\Delta^{\perp} H+\operatorname{tr} B\left(\cdot, A_{H} \cdot\right)-(m-1) H=0, \\
\frac{m}{2} \operatorname{grad}|H|^{2}+2 \operatorname{tr} A_{\nabla^{\perp} H}(\cdot)+\operatorname{tr}(f) F H=0,
\end{array}\right.
$$

where all terms are zero in the second equality. This concludes the proof.

When $M$ is a hypersurface, the characterization of biharmonicity can be expressed in a simpler way. Namely, for biharmonic hypersurfaces with $F H$ tangent to $M$, we have the following result.

Proposition 3.4. Let $M$ a hypersurface of $\mathbb{S}^{p} \times \mathbb{S}^{n-p}$ with non-zero constant mean curvature and such that $F H$ is tangent to $M$. Then $M$ is biharmonic if and only if

$$
\operatorname{tr}(f)=0 \quad \text { and } \quad|B|^{2}=(n-2) .
$$

Proof. This is a direct consequence of Corollary 3.2. When $M$ is a hypersurface of non-zero constant mean curvature with $F H$ tangent to $M$, the first equation becomes $\operatorname{tr}\left(B\left(\cdot, A_{H} \cdot\right)\right)-(n-2) H=0$, that is, $|B|^{2}=(n-2)$ since $\operatorname{tr}\left(B\left(\cdot, A_{H} \cdot\right)\right)=|B|^{2} H$. The second equation of assertion (1) in Corollary 3.2 is reduced to $\operatorname{tr}(f)=0$.

Using this proposition, we can compute the scalar curvature of biharmonic hypersurfaces with $F H$ tangent to $M$.

Proposition 3.5. Let $M$ be a proper-biharmonic hypersurface of $\mathbb{S}^{p} \times \mathbb{S}^{n-p}$ with non-zero constant mean curvature and such that $F H$ is tangent to $M$. Then the scalar curvature of $M$ is constant and given by

$$
\operatorname{Scal}_{M}=(n-2)(n-3)+(n-1)^{2}|H|^{2} .
$$

Proof. First, by the Gauss equation, we have

$$
\operatorname{Scal}_{M}=\sum_{i, j=1}^{n-1}\left\langle\widetilde{R}\left(X_{i}, X_{j}\right) X_{j}, X_{i}\right\rangle-|B|^{2}+(n-1) H^{2} .
$$

We recall that the curvature $\widetilde{R}$ of $\mathbb{S}^{p} \times \mathbb{S}^{n-p}$ is given by (4) with $a=1$ and $b=0$. Hence, we have

$$
\left\langle\widetilde{R}\left(X_{i}, X_{j}\right) X_{j}, X_{i}\right\rangle=1-\left\langle X_{i}, X_{j}\right\rangle^{2}+\left\langle f X_{i}, X_{i}\right\rangle\left\langle f X_{j}, X_{j}\right\rangle-\left\langle f X_{i}, X_{j}\right\rangle^{2} .
$$


By summation, we get

$$
\sum_{i, j=1}^{n-1}\left\langle\widetilde{R}\left(X_{i}, X_{j}\right) X_{j}, X_{i}\right\rangle=(n-1)(n-2)+\operatorname{tr}(f)^{2}-\sum_{i, j=1}^{n-1}\left\langle f X_{i}, X_{j}\right\rangle^{2} .
$$

Since $f$ is symmetric and using (5), we have

$$
\begin{aligned}
\sum_{i, j=1}^{n-1}\left\langle f X_{i}, X_{j}\right\rangle^{2} & =\sum_{j=1}^{n-1}\left\langle f X_{j}, f X_{j}\right\rangle \\
& =\sum_{j=1}^{n-1}\left\langle F X_{j}, F X_{j}\right\rangle-\left\langle X_{j}, F \nu\right\rangle^{2} \\
& =(n-1)-|s \nu|^{2} .
\end{aligned}
$$

Now, we will use the hypothesis that $F H$ is tangent to $M$. Since $M$ is a hypersurface, this is equivalent to the fact that $F \nu$ is tangent to $M$, where $\nu=\frac{H}{|H|}$ is the unit normal to the hypersurface. Then this implies that $s \nu=F \nu$ and therefore $|s \nu|=1$. Hence, we have

$$
\sum_{i, j=1}^{n-1}\left\langle f X_{i}, X_{j}\right\rangle^{2}=n-2
$$

Finally, since by the previous Proposition, we have $\operatorname{tr}(f)=0$ and $|B|^{2}=$ $(n-2)$, we get

$$
\begin{aligned}
\operatorname{Scal}_{M} & =(n-1)(n-2)+\operatorname{tr}(f)^{2}-(n-2)-|B|^{2}-(n-1)^{2}|H|^{2} \\
& =(n-2)(n-3)+(n-1)^{2}|H|^{2}
\end{aligned}
$$

Remark 3.6. One could obtain results comparable to Corollary 3.3, Propostion 3.4 and 3.5 for submanifolds with FH normal. However, all the estimates would depend on $\operatorname{tr}(f)$. We will not write these results here.

Finally, we give a non-existence result in the case of the product of a sphere with a hyperbolic space. We recall that in this case, we have $a=0$ and $b=1$.

Proposition 3.7. There exists no proper-biharmonic submanifolds of $\mathbb{S}^{p} \times$ $\mathbb{H}^{n-p}$ of dimension $m \geqslant 2$ with parallel mean curvature and such that $F H$ is not normal.

Proof. First, we remark that since $H$ is parallel and not identically zero $(M$ is proper-biharmonic), then $H$ has no zeros. Moreover, $F$ is an isometry, then $F H$ also has no zeros. If $M$ is biharmonic, by Theorem 3.1, we have

$$
\frac{m}{2} \operatorname{grad}|H|^{2}+2 \operatorname{tr} A_{\nabla^{\perp} H}(\cdot)-(m-1) s H=0 .
$$


If $M$ has parallel mean curvature, then this equation becomes $s H=0$. If, in addition, $F H$ is not normal, then its tangential part $s H$ does not vanish identically, which is impossible.

Remark 3.8. In particular, there exists no parallel mean curvature properbiharmonic submanifolds of $\mathbb{S}^{p} \times \mathbb{H}^{n-p}$ such that $F H$ is tangent.

\section{References}

[1] Balmuş A., Montaldo S. and Oniciuc C.: Biharmonic PNMC submanifolds in spheres. Ark. Mat. (in press)

[2] Caddeo R., Montaldo S., Oniciuc C.: Biharmonic submanifolds in spheres. Israel J. Math. 130, 109-123 (2002)

[3] Chen B.Y.: Total Mean Curvature and Submanifolds of Finite Type. Series in Pure Mathematics 1. World Scientific Publishing Co., Singapore (1984)

[4] Eells J. and Sampson J.H.: Harmonic mappings of Riemannian manifolds. Amer. J. Math. 86, 109-160 (1964)

[5] Fetcu D., Loubeau E., Montaldo S. and Oniciuc C.: Biharmonic submanifolds of $\mathbb{C} P^{n}$. Math. Z. 266, 505-531 (2010)

[6] Fetcu D. and Oniciuc C.: Explicit formulas for biharmonic submanifolds in Sasakian space forms. Pacific J. Math. 240 (1), 85-107 (2009)

[7] D. Fetcu, C. Oniciuc and H. Rosenberg, biharmonic submanifolds with parallel mean curvature in $\mathbb{S}^{n} \times \mathbb{R}$, J. Geom. Anal. (in press)

[8] Jiang G.Y.: 2-harmonic maps and their first and second variational formulas, Chinese Ann. Math. Ser. A 7 (4), 389-402 (1986)

\section{Julien ROTH}

(J. Roth) LAMA, Université Paris-Est Marne-la-Vallée, Cité Descartes, Champs sur Marne, 77454 Marne-la-Vallée cedex 2, France

e-mail: julien.roth@univ-mlv.fr 